\title{
Drenaje biliar guiado por ultrasonografía endoscópica en la obstrucción biliar maligna, experiencia inicial en un centro de Argentina. Serie de casos
}

\author{
Augusto Villaverde, Juan Eduardo Pizzala, Inés Cecilia Oria, Dante Manazzoni, Víctor Abecia, \\ Manuel Alejandro Mahler, Dalila Urgiles, Mariano Marcolongo
}

Servicio de Gastroenterología, Hospital Italiano de Buenos Aires. Ciudad Autónoma de Buenos Aires, Argentina.

Acta Gastroenterol Latinoam 2020;50(3):279-284

Recibido: 08/06/2020 / Aceptado: 07/08/2020 / Publicado online: 28/09/2020

\section{Resumen}

En los pacientes en que fracasa o no es factible la realización de la colangiopancreatografía retrógrada endoscópica para drenaje de la vía biliar por ictericia obstructiva, el drenaje biliar guiado por ultrasonografía endoscópica es una opción viable. Esta técnica de drenaje presenta una menor tasa de complicaciones y una mejor calidad de vida con respecto al drenaje percutáneo o quirúrgico. El objetivo de este trabajo es presentar nuestra experiencia inicial con esta técnica en pacientes con estenosis maligna de la vía biliar, en un centro de derivación. Se realizó un estudio descriptivo de una serie de casos, en el que se incluyeron cinco pacientes con ictericia obstructiva de origen neoplásico en los que no fue factible realizar drenaje por colangiopancreatografía retrógrada endoscópica y a los que se les efectuó drenaje biliar guiado por ultrasonografía endoscópica. Se observó un éxito técnico en el

Correspondencia: Augusto, Villaverde

Perón 4190 (C.P.: 1199). Ciudad Autónoma de Buenos Aires, Argentina Tel.: +549-2215925737

Correo electrónico: augusto.villaverde@hospitalitaliano.org.ar
$100 \%$ de los casos y un éxito clínico en el $80 \%$ de los casos. Registramos una complicación temprana por sangrado, y dos complicaciones tardias por infección y migración de prótesis, siendo todas resueltas con tratamiento conservador. No se registraron eventos adversos graves. El drenaje biliar guiado por ultrasonografía endoscópica es una modalidad terapéutica viable y efectiva en casos seleccionados, con una alta tasa de éxito técnico y clínico, particularmente en cuadros de obstrucción biliar maligna. Las complicaciones suelen ser leves y resolverse de forma conservadora.

Palabras claves. Drenaje biliar, ultrasonido endoscópico, drenaje biliar guiado por ultrasonografía endoscópica.

\section{EUS-Guided biliary drainage for malignant biliary obstruction, initial experience in a centre in Argentina. Case series}

\section{Summary}

When endoscopic retrograde cholangiopancreatography (ERCP) fails or is not feasible for drainage of the bile duct, endoscopic ultrasound-guided biliary drainage (EUS-BD) is a viable option. This technique of drainage has a lower complication rate and a better quality of life concerning percutaneous or surgical drainage. The aim of this work is to show our initial experience in this technique, in patients with malignant bile duct stenosis, in a referral centre. We performed a descriptive study of case series. Five patients with obstruc- 
tive jaundice of neoplastic origin, in whom ERCP drainage was not feasible, were included EUS-BD was performed. Technical success of $100 \%$ was achieved in all cases and clinical success in four cases (80\%). Post-procedural bleeding occurred in one patient, and two late complications: infection and prosthetic migration, all of which were resolved with conservative treatment. No serious adverse events were recorded. EUS-BD is a feasible and effective treatment option in selected casesparticularly in cases of malignant biliary obstruction, with high technical and clinical success rates. Complications are usually mild and resolve conservatively.

Key words. Biliary drainage, endoscopic ultrasound, endoscopic ultrasonography-guided biliary drainage.

\section{Abreviaturas}

CPRE: Colangiopancreatografía retrógrada endoscópica. DB-USE: Drenaje biliar guiado por ultrasonografía endoscópica.

CDS-USE: Coledocoduodenostomía guiada por ultrasonografía endoscópica.

HGS-USE: Hepático gastrostomia guiada por ultrasonografía endoscópica.

USE: Ultrasonido endoscópico.

\section{Introducción}

En los casos de obstrucción biliar maligna, la mayoría de los pacientes son diagnosticados en estadios avanzados, no siendo candidatos a un tratamiento quirúrgico con intención curativa. Independientemente de la etiología, el drenaje de la vía biliar para la resolución de la ictericia y la mejoría en la calidad de vida es un procedimiento paliativo realizado de forma habitual.

La CPRE es el procedimiento de elección para el drenaje biliar; sin embargo, en los casos en que este no es factible, el DB-USE es una opción válida, que proporciona múltiples ventajas respecto del drenaje percutáneo o quirúrgico. ${ }^{1-3}$

En múltiples estudios se ha demostrado la seguridad y viabilidad de DB-USE; sin embargo, esta técnica no está exenta de complicaciones, en ocasiones graves, por lo que es recomendable que sea realizada por expertos y en centros de complejidad. $7,10,11$

Dependiendo de la vía de acceso (intrahepática o extrahepática), puede realizarse el DB-USE por la ruta transmural o transpapilar, con sus diversas variantes.

A continuación describimos cinco casos, en los que se optó por DB-USE ante el fallo o la imposibilidad de realizar CPRE por obstrucción de vía biliar de etiología maligna. Mostramos nuestra experiencia inicial, en el período comprendido desde el 2019 al 2020.

\section{Métodos}

Estudio descriptivo de una serie de casos. Se analizaron cinco casos de drenaje biliar guiado por ultrasonografía endoscópica ante el fallo o la imposibilidad de realizar CPRE, en un período comprendido entre enero del 2019 hasta mayo del 2020 en el servicio de gastroenterología del Hospital Italiano de Buenos Aires.

Las variables estudiadas fueron: éxito técnico (definido como el adecuado posicionamiento de la prótesis, al menos $1 \mathrm{~cm}$ por arriba y por debajo de la obstrucción con adecuada salida del medio de contraste), éxito clínico (definido como el descenso de al menos el 50\% de los valores iniciales de bilirrubina en las tres semanas posteriores al procedimiento, sin necesidad de cirugía de rescate), etiología, complicaciones inmediatas (si se presentaron en menos de 24 horas del procedimiento) y complicaciones tardías (si se presentaron posteriormente a las 24 horas del procedimiento).

Los síntomas que motivaron el estudio fueron la ictericia obstructiva en cuatro casos y la colangitis en uno. Todos los casos tenían una imagen abdominal que mostraba hallazgos compatibles con obstrucción de vía biliar de etiología tumoral.

El procedimiento terapéutico se realizó en el quirófano, por un endoscopista operador y enfermería calificada. En todos los casos se empleó sedación profunda en perfusión con propofol al 1\%, con intubación orotraqueal, asociada a oxigenoterapia y monitorización no invasiva realizada por médico anestesiólogo. Se utilizó un procesador Olympus EU-ME1 con ecoendoscopio lineal Olympus (GF-UC140P-AL5) e insuflación con bomba de $\mathrm{CO}_{2}$.

En todos los casos se colocaron endoprótesis biliares metálicas parcialmente cubiertas de $10 \mathrm{~mm}$ de diámetro y longitud variable (Wallflex, Boston Scientific).

En cuatro casos se alcanzó el bulbo duodenal para la localización y la visualización del colédoco dilatado, y se realizó drenaje biliar transmural por coledocoduodenostomía (Figura 1, Video 1); en el caso restante, desde el estómago se valoró la vía biliar intrahepática y se realizó drenaje biliar anterógrado transhepático transpapilar (Figura 2, Video 2). Los procedimientos se realizaron guiados por radiología, ecoendoscopía y visión endoscópica directa. Finalizado el procedimiento, el paciente fue trasladado a la unidad de hospitalización. 
Figura 1. Secuencia de drenaje biliar ecoguiado mediante técnica de coledocoduodenostomía

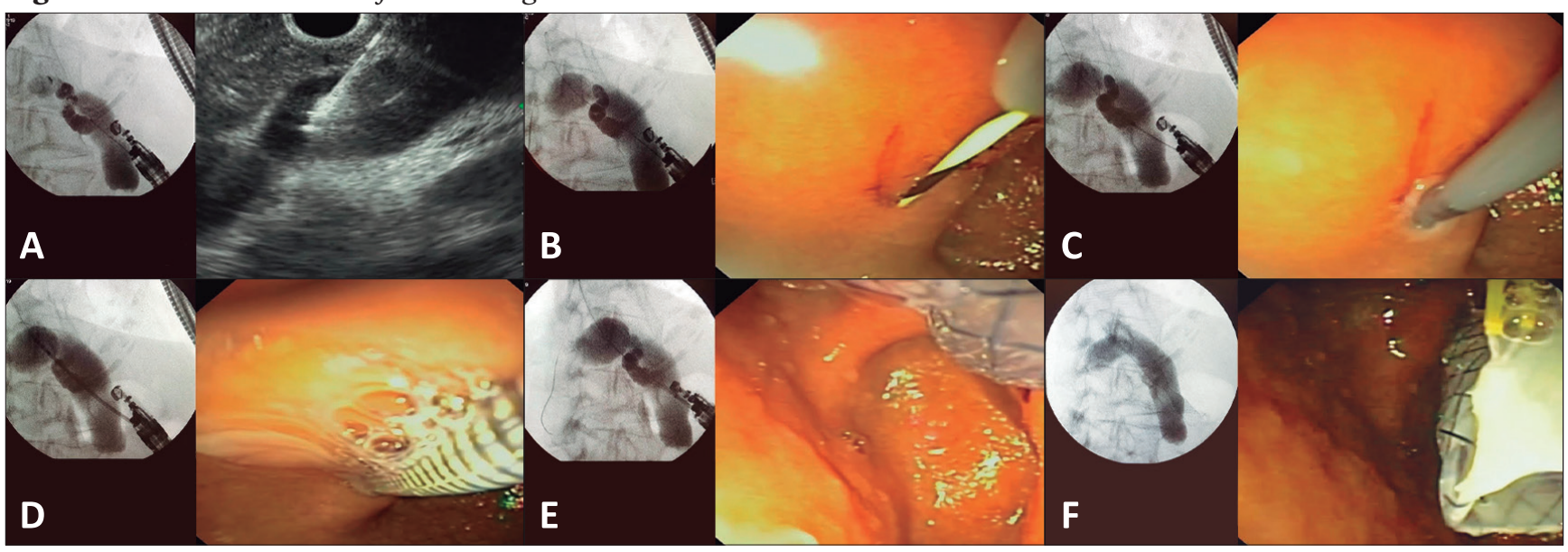

A) Se realiza punción del colédoco a través de bulbo duodenal. B) Se ingresa guía hidrofilica a través de aguja de punción. C) se dilata trayecto con dilatador de Soehendra. D) Se ingresa prótesis metálica parcialmente cubierta. E) Se libera prótesis bajo control radioscópico y endoscópico. F) Se documenta correcto posicionamiento de la prótesis y drenaje de material purulento a través de la misma.

Video 1. Se muestra video de drenaje biliar ecoguiado transmural mediante técnica de coledocoduodenostomía Visualizar Videos en nuestra web http:/lactagastro.org/

Figura 2. Secuencia de drenaje biliar ecoguiado transpapilar anterógrado

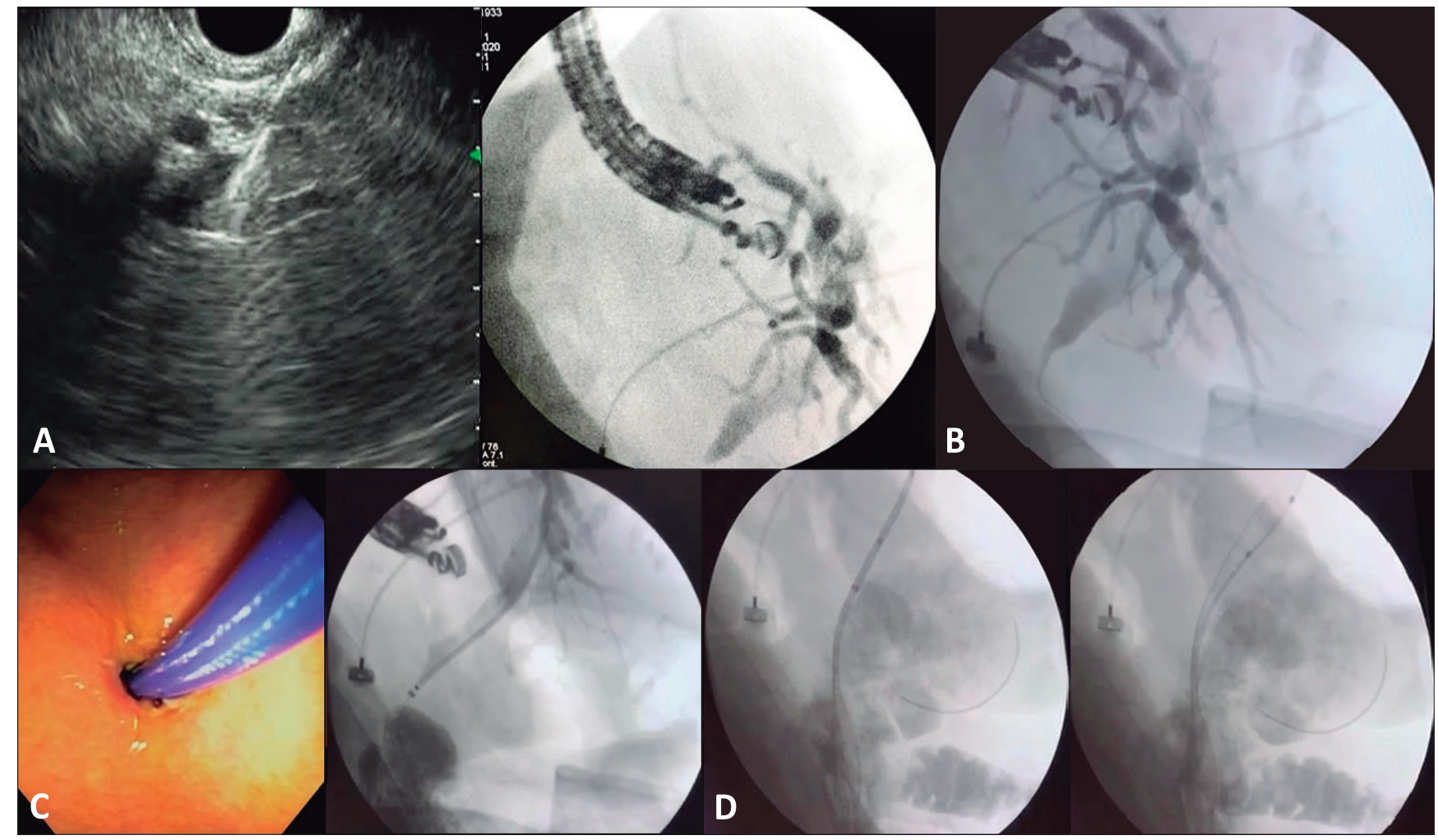

A) Se realiza punción de vía biliar intrahepática a través de curvatura menor gástrica. B) Se pasa guía hidrofílica a través de papila duodenal por vía anterógrada. C) Se pasa prótesis metálica a través de guía, previa dilatación del trayecto con cistotomo de 10 french. D) Se libera prótesis bajo guía radioscópica.

Video 2. Se muestra video de drenaje biliar ecoguiado transpapilar anterógrado

Visualizar Videos en nuestra web http:/lactagastro.org/ 
Se usó para la captura de datos la historia clínica electrónica institucional del Hospital Italiano de Buenos Aires, en la cual todos los pacientes al momento de empadronarse han firmado de forma voluntaria y certificada el consentimiento informado para la utilización de sus datos. Todos los datos fueron tratados con máxima confidencialidad y de manera anónima, con acceso restringido solo para el personal autorizado a los fines de este estudio. El trabajo fue realizado bajo el protocolo de ética del hospital para estos casos.

\section{Resultados}

Se incluyeron cinco pacientes, con una edad media de 78 años. Todas las causas de obstrucción biliar fueron de etiología tumoral, en pacientes no candidatos a tratamiento quirúrgico. Las características clínicas y demográficas de los pacientes se resumen en la Tabla 1. La mediana del diámetro del conducto biliar común fue $16 \mathrm{~mm}(15-16)$.

En cuatro pacientes se realizó drenaje transmural por coledocoduodenostomía guiada por ultrasonografía endoscópica (CDS-USE), y en un paciente se realizó drenaje anterógrado transpapilar por hepático gastrostomía guiada por ultrasonografía endoscópica (HGS-USE). En tres pacientes se colocaron endoprótesis biliares metálicas autoexpandibles parcialmente cubiertas de 10 x $80 \mathrm{~mm}$ y en los dos restantes de 10 x $60 \mathrm{~mm}$.

Todos los pacientes presentaron ictericia obstructiva, con una mediana de bilirrubina basal de $16 \mathrm{mg} / \mathrm{dl}$ (6-15), bilirrubina a la semana del procedimiento de $3,1 \mathrm{mg} / \mathrm{dl}$ $(3,5-4)$ y bilirrubina a la tercera semana del procedimiento de $1 \mathrm{mg} / \mathrm{dl}(0,8-2,6)$.

Tabla 1. Características clínicas y demográficas de los pacientes

\begin{tabular}{|c|c|c|c|c|c|c|c|c|c|c|c|}
\hline Paciente & $\begin{array}{l}\text { Edad } \\
\text { (años) }\end{array}$ & Sexo & $\begin{array}{c}\text { Causa de } \\
\text { fallo } 0 \\
\text { imposibilidad } \\
\text { de CPRE }\end{array}$ & $\begin{array}{c}\text { Etiología } \\
\text { de la } \\
\text { obstrucción } \\
\text { biliar }\end{array}$ & $\begin{array}{l}\text { Diámetro } \\
\text { del CBC } \\
(\mathrm{mm})\end{array}$ & $\begin{array}{c}\text { Tiempo del } \\
\text { procedimiento } \\
\text { (min) }\end{array}$ & $\begin{array}{c}\text { Días de } \\
\text { hospitalización } \\
\text { post } \\
\text { procedimiento }\end{array}$ & $\begin{array}{l}\text { Éxito } \\
\text { técnico }\end{array}$ & $\begin{array}{l}\text { Éxito } \\
\text { clínico }\end{array}$ & $\begin{array}{c}\text { Complicaciones } \\
\text { tempranas }\end{array}$ & $\begin{array}{c}\text { Complicaciones } \\
\text { tardías }\end{array}$ \\
\hline$N^{\circ} 1$ & 78 & $\mathrm{~F}$ & ED & Ca páncreas & 16 & 34 & 4 & Sí & Sí & No & No \\
\hline$N^{\circ} 2$ & 55 & M & ED & $\begin{array}{l}\text { Ca ampolla } \\
\text { Vater }\end{array}$ & 10 & 55 & 4 & Sí & No & No & $\begin{array}{c}\text { Sí } \\
\text { (colangitis) }\end{array}$ \\
\hline$N^{\circ} 3$ & 59 & M & $\mathbb{P}$ & Ca páncreas & 16 & 75 & 2 & Sí & Sí & $\begin{array}{c}\text { Sí } \\
\text { (hemorragia) }\end{array}$ & $\begin{array}{c}\text { Sí } \\
\text { (migración } \\
\text { de prótesis) }\end{array}$ \\
\hline$N^{\circ} 4$ & 87 & $F$ & $\mathbb{P}$ & Ca páncreas & 15 & 48 & 8 & Sí & Sí & No & No \\
\hline$N^{\circ} 5$ & 86 & $M$ & ED & Ca páncreas & 16 & 42 & 3 & Sí & Sí & No & No \\
\hline
\end{tabular}

CPRE: colangiopancreatografía retrógrada endoscópica; ED: estenosis duodenal; IP: infiltración de la papila; CBC: conducto biliar común.

Se observó un éxito clínico en cuatro casos y un paciente requirió una cirugía de rescate con hepaticoyeyuno anastomosis antes de las tres semanas, por falta de descenso de bilirrubina y colangitis recurrente. El éxito técnico se obtuvo en todos los casos.

La mediana de duración del procedimiento fue de 48 minutos (42-55), con una mediana de estancia hospitalaria posterior al procedimiento de 4 días (3-4).
En un paciente se observó un evento adverso intraprocedimiento (hemorragia, la cual se limitó al expandirse la prótesis implantada a través del trayecto fistuloso). Se registraron dos eventos adversos tardíos (colangitis y una migración de la prótesis que fue resuelta endoscópicamente con colocación de una nueva prótesis a través del trayecto fistuloso generado) (Figura 3). No se observaron complicaciones graves. 
Figura 3. Secuencia de resolución endoscópica de complicación tardía (migración)

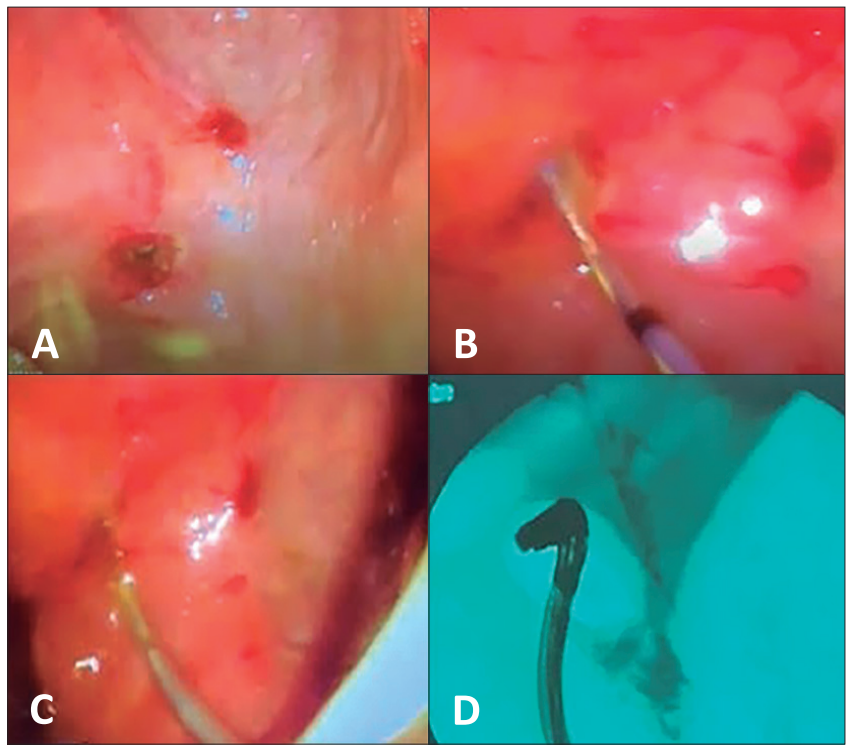

A) Se observa orificio fistuloso en bulbo. B) Se canula trayecto fistuloso con papilotomo y se confirma localización biliar por colangiografía. C) Se progresa prótesis metálica a través de guía. D) Se libera prótesis a través de fístula coledocoduodenal.

\section{Discusión}

La CPRE es el procedimiento de elección para el drenaje biliar, tanto de obstrucciones biliares benignas como malignas. Sin embargo, puede fallar en un $4 \%$ a $16 \%$ de los casos debido a inexperiencia del operador, una anatomía alterada quirúrgicamente, patología ampular u obstrucción maligna. ${ }^{1}$

En la última década, el papel del DB-USE surgió como una opción de rescate después de una CPRE fallida. En un análisis de cohorte retrospectivo, se comparó el drenaje percutáneo con DB-USE después de una CPRE fallida, y se observó una menor tasa de eventos adversos, menos intervenciones y menores costos asociado al DB-USE. ${ }^{2,3}$

Dos recientes ensayos aleatorios compararon el drenaje biliar transmural guiado por USE versus CPRE para tratamiento primario de la obstrucción biliar maligna, e informaron tasas de éxito y eventos adversos similares. ${ }^{4,5}$

Dentro de las indicaciones para realizar una DB-USE se incluyen: CPRE convencional fallida, anatomía alterada, tumor que impide el acceso al árbol biliar y contraindicación al acceso percutáneo.

El DB-USE representa un procedimiento complejo, que requiere endoscopistas expertos, con una buena curva de aprendizaje, para que se desarrolle efectivamente. ${ }^{6}$

Dentro de las técnicas de drenaje biliar más comu- nes está la transmural, en la cual se accede a la vía biliar intrahepática o extrahepática, a través de la pared duodenal (CDS-USE) o gástrica (HGS-USE), generando una fístula entre la vía biliar y el sitio de acceso; el drenaje anterógrado se caracteriza por acceder a la vía biliar progresando la prótesis por vía anterógrada a través de la papila (drenaje transpapilar); otra modalidad es la técnica de rendez-vous en la cual se progresa una guía en forma anterógrada hacia la papila guiada por USE, y posteriormente se canaliza la vía biliar con el duodenoscopio completando el drenaje. En este caso es necesario tener una anatomía duodenal viable. ${ }^{6-8}$

En la actualidad no hay consenso sobre cuál es la mejor vía de acceso y drenaje, sin embargo, presentan diferentes ventajas y limitaciones de acuerdo al caso. ${ }^{11}$

En lo reportado, independientemente del enfoque, la tasa de éxito acumulada es de $84 \%$ a $93 \%$ y la tasa de eventos adversos de $23 \%$.

Las complicaciones asociadas al DB-USE incluyen infección (peritonitis, colangitis, colecistitis), pancreatitis, neumoperitoneo, fuga biliar / biloma, sangrado, dolor abdominal, y migración del stent. ${ }^{7}$

La infección es la complicación más común $(2,1 \%$ a $3,5 \%)$, seguida de fuga biliar y migración del stent. ${ }^{9}$

Dentro de las opciones de prótesis biliares para DB-USE se encuentran los stent plásticos (doble pigtail), los stent metálicos (cubiertos y parcialmente cubiertos), y recientemente se han desarrollado los LAMS para derivaciones biliares y una prótesis autoexpandible especialmente diseñada para derivación hepaticogástrica (estas dos últimas no disponibles para su uso en la Argentina).

En este trabajo, los stent que utilizamos fueron los stent metálicos parcialmente cubiertos, a fin de evitar la fuga de bilis y la migración; la longitud de los mismos fue determinada por la extensión de la estenosis biliar, con un diámetro estándar de $10 \mathrm{~mm}$.

En nuestro estudio, se registró una hemorragia intraprocedimiento en un paciente con tumor de páncreas con infiltración de pared de segunda porción duodenal, posterior a realizar punción desde bulbo con aguja de $19 \mathrm{G}$ y pasar guía 0,035 sobre vía biliar dilatada, al ingresar con cistostomo de 10 french a través de la pared observándose sangrado en napa, que luego de liberar prótesis metálica parcialmente cubierta de $60 \times 10 \mathrm{~mm}$ en el trayecto fistuloso produjo hemostasia mecánica con cese de sangrado. Este mismo paciente presentó como complicación tardía migración de la prótesis metálica hacia la luz duodenal, la cual se extrajo con pinza de cuerpo extraño. Posteriormente se canuló con esfinterótomo y alambre guía a través de la fístula, contrastando la vía biliar de forma selectiva, sin imágenes de defecto en su interior, y se colocó 
un nuevo stent biliar parcialmente cubierto de $8 \mathrm{~cm}$, con adecuado posicionamiento y expansión de la prótesis. En el mismo procedimiento por estenosis a nivel de la segunda porción duodenal se colocó stent duodenal.

La colangitis recurrente fue otra complicación tardía que fue observada en otro paciente, manejada con tratamiento antibiótico y requerimiento posterior de derivación biliodigestiva.

Estas complicaciones se encuentran dentro de las reportadas en la literatura mundial y quizás una de las explicaciones es que no existen dispositivos (excepto los nuevos LAMS diseñados para coledocoduodenoanastomosis) específicamente diseñados para estos procedimientos, sino que los que se utilizan son los mismos que se usan para intervencionismo biliar transpapilar convencional.

La presente serie de casos, a pesar de ser limitada por el número de pacientes, muestra nuestra experiencia inicial con una técnica segura y efectiva para el tratamiento de la obstrucción biliar maligna, que, progresivamente, se está convirtiendo en nuestra práctica diaria en una opción terapéutica válida en casos seleccionados.

Sostén financiero. Este documento no recibió ningún tipo de financiación o patrocinio externo.

\section{Referencias}

1. Holt BA, Hawes R, Hasan M, Canipe A, Tharian B, Navaneethan U, Varadarajulu S. Biliary drainage: role of EUS guidance. Gastrointestinal Endoscopy 2016; 83 (1): 160-165.

2. Bliss LA, Eskander MF, Kent TS, Watkins AA, de Geus SW, Storino A, Ng SC, Callery MP, Moser AJ, Tseng JF. Early surgical bypass versus endoscopic stent placement in pancreatic cancer. HPB (Oxford) 2016; 18 (8): 671-677.
3. Khashab MA, Valeshabad AK, Afghani E, Singh VK, Kumbhari V, Messallam A, Saxena P, El Zein M, Lennon AM, Canto MI, Kalloo AN. A comparative evaluation of EUS-guided biliary drainage and percutaneous drainage in patients with distal malignant biliary obstruction and failed ERCP. Dig Dis Sci 2015; 60 (2): 557-565.

4. Bang JY, Navaneethan U, Hasan M, Hawes R, Varadarajulu S. Stent placement by EUS or ERCP for primary biliary decompression in pancreatic cancer: a randomized trial (with videos). Gastrointestinal Endoscopy 2018; 88 (1): 9-17.

5. Paik WH, Lee TH, Park DH, Choi JH, Kim SO, Jang S, Kim DU, Shim JH, Song TJ, Lee SS, Seo DW, Lee SK, Kim MH. EUS-guided biliary drainage versus ERCP for the primary palliation of malignant biliary obstruction: a multicenter randomized clinical trial. Am J Gastroenterology 2018; 113 (7): 987.

6. Kahaleh M, Artifon EL, Pérez Miranda M, Gaidhane M, Rondon C, Itoi T, Giovannini M. Endoscopic ultrasonography guided drainage: summary of consortium meeting, May 21, 2012, San Diego, California. World J Gastroenterology 2015; 21 (3): 726-741.

7. Kedia P, Gaidhane M, Kahaleh M. Endoscopic guided biliary drainage: how can we achieve efficient biliary drainage? Clinical Endoscopy 2013; 46: 543-551.

8. Sarkaria S, Lee HS, Gaidhane M, Kahaleh M. Advances in endoscopic ultrasound-guided biliary drainage: a comprehensive review. Gut Liver 2013; 7: 129-136.

9. Sharaiha RZ, Khan MA, Kamal F, Tyberg A, Tombazzi CR, Ali B, Tombazzi C, Kahaleh M. Efficacy and safety of EUS-guided biliary drainage in comparison with percutaneous biliary drainage when ERCP fails: a systematic review and meta-analysis. Gastrointestinal Endoscopy 2017; 85 (5): 904-914.

10. Gupta K, Pérez-Miranda M, Kahaleh M, Artifon EL, Itoi T, Freeman ML, De-Serna C, Sauer B, Giovannini M. Endoscopic ultrasound-assisted bile duct access and drainage and complications of a technique in evolution. J Clin Gastroenterology 2014; 48 (1): 80-87.

11. Wang K, Zhu J, Xing L, Wang Y, Jin Z, Li Z. Assessment of efficacy and safety of EUS-guided biliary drainage: a systematic review. Gastrointestinal Endoscopy 2016; 83 (6): 1218-1227. 